Proyecciones

Vol. 18, No 1, pp. 107-126, July 1999

Universidad Católica del Norte

Antofagasta - Chile

\title{
CHAMPS DE VECTEURS HOLOMORPHES TANGENTS AUX HYPERSURFACES POLYNÔMIALES RIGIDES DE $\mathbb{C}^{2}$
}

\author{
ABDELAZIZ CHAOUECH \\ Universite Mouloy Ismail, Errachidia-Maroc
}

\begin{abstract}
In this paper, we present two works. The first give a complet description of tangent holomorphic vector fields of rigid polynomial hypersurfaces in $\mathbf{C}^{2}$ which is not spheric at the origine.

In the second we studie the propre holomorphic mappings between rigid polynomial domains $\Omega_{1}, \Omega_{2}$ in $\mathbf{C}^{2}$, more precisely we prove that, if $b \Omega_{1}$ is strictely pseudo-convexe and not spheric in at less one point, then such map is of the form $\left(\alpha w+f_{1}(z), f_{2}(z)\right)$, where $\alpha \in R^{*}$ and $f_{1}, f_{2}$ are two polynômes.
\end{abstract}




\section{Introduction et résultats}

On dit d'une hypersurface analytique réelle $M$ de $\mathbf{C}^{2}$ qu'elle est rigide si elle peut être décrite par une équation de la forme $r(w, \bar{w}, z, \bar{z})=: \operatorname{Im} w+$ $F(z, \bar{z})=0$ où $F$ est une fonction analytique réelle telle que $F(0,0)=$ $\frac{\partial F}{\partial z}(0,0)=0$. Les champs de vecteurs holomorphes tangents à $M$ sont les champs de la forme $X=X_{1} \frac{\partial}{\partial w}+X_{2} \frac{\partial}{\partial z}$ où les fonctions $X_{1}$ et $X_{2}$ sont holomorphes au voisinage de l'origine et tels que $R e\left[X_{1} \frac{\partial r}{\partial w}+X_{2} \frac{\partial r}{\partial z}\right]$ soit identiquement nul sur $M$. L'ensemble $\mathcal{L}(M)$ de ces champs a une structure d'algèbre de Lie pour le crochet usuel et l'on sait depuis les travaux d'E. Cartan que cette algèbre est de dimension finie dès que $M$ est non dégénérée à l'origine c'est à dire dès que $\Delta r(0,0) \neq 0$ (en fait la rigidité est superflue). Plus précisément, si la dimension réelle de $\mathcal{L}(\Lambda)$ est supérieure ou égale à 4 alors $M$ est sphérique à l'origine, ce qui signifie qu'un biholomorphisme local applique $M$ sur la sphère euclidienne. Une démonstration de ce résultat est accessible dans l'article de $N$. Stanton ([5]) où l'on trouvera également une étude détaillée de l'algèbre $\mathcal{L}(M I)$ dans le cas dégénéré. On peut d'autant plus facilement expliciter $\mathcal{L}(M)$ que sa dimension est petite et la fonction $F$ simple. L'objet de cet article est de traiter le cas polynômial. Notre principal résultat est le suivant:

\section{Théorème 1 :}

Soit $P(z, \bar{z})$ un polynôme sans termes harmoniques tel que $\triangle P(0,0) \neq$ 0 .

Si l'hypersurface $M=:\{\operatorname{Imw}+P(z, \bar{z})=0\}$ est non sphérique en $(0,0)$ alors les champs de vecteurs holomorphes tangents à $M$ Isont de l'une des formes suivantes:

(i) $X=\lambda \frac{\partial}{\partial w}$

(ii) $X=\left(\alpha w+A_{1}(z)\right) \frac{\partial}{\partial w}+A_{2}(z) \frac{\partial}{\partial z}$

(iii) $X=\left(\alpha w^{2}+A_{1}(z) w+A_{2}(z)\right) \frac{\partial}{\partial w}+\left(A_{2}(z) w+B_{2}(z)\right) \frac{\partial}{\partial z}$

où $\lambda, \alpha \in \mathbb{R}$ et les $A_{j}, B_{j}$ sont des fractions rationnelles telles que $A_{2} \neq \equiv$ et $\operatorname{deg}\left(B_{2}\right) \leq \operatorname{deg} P$.

Notre étude donne en fait les fractions $A_{j}$ et $B_{j}$ sous forme explicite, en vue des applications, nous avons systématiquement investigué cet aspect pour le cas des champs polynômiaux. Nous établissons la: 
Proposition 1 : Soit $M=\{\operatorname{Im} w+P(z, \bar{z})=0\}$ une hypersurface polynômiale rigide telle que $\triangle P \geq 0$ et $\triangle P(0,0) \neq 0$ soit $\vec{X}$ un champ de vecteurs holomorphe défini au voisinage de l'origine de $\mathbf{C}^{2}$ et tangent à $M$ dont on suppose qu'il est de l'une des formes (ii) ou (iii) du théorème 1. .

Si $\vec{X}$ est de la forme (ii) où $\alpha \in \mathbb{R}$ et $A_{1}, A_{2}$ sont des polynômes tels que $A_{2} \neq 0$, alors on se trouve dans l'un des cas suivants:

a) $\alpha \neq 0, A_{2}=a_{0}^{2}+a_{1}^{2} z,\left(a_{0} \in \mathbf{C}, a_{1} \in \mathbf{C}^{*}\right)$ et il existe un polynôme holomorphe $h$ tel que le changementcle variables $\left(w^{*}, z^{*}\right)=\left(w+h(z), a_{0}^{2}+\right.$ $\left.a_{1}^{2} z\right)$ transforme $M$ en $M^{*}=\left\{I m w^{*}+H^{*}\left(z^{*}, \bar{z}^{*}\right)=0\right\}$

où $H^{*}$ est un polynôme homogène. De plus $H^{*}=M\left|z^{*}\right|^{2 m}$ si $I m a_{1}^{2} \neq 0$. b) $\alpha=0, A_{2}=a_{0}^{2}+i \lambda z,\left(a_{0}^{2} \in \mathbf{C}, \lambda \in \mathbb{R}^{*}\right)$ et il existe un polynôme holomorphe $h$ tel que le changement de variables $\left(w^{*}, z^{*}\right)=\left(w+h(z), a_{0}^{2}+\right.$ $i \lambda z$ ) transforme $M$

$$
\text { en } M^{*}=\left\{I m w^{*}+\sum_{k=1}^{N} \alpha_{k}\left|z^{*}\right|^{2 k}=0\right\} \text { et } \vec{X} \text { en } \lambda_{1} \frac{\partial}{\partial w^{*}}+i \lambda_{2} z^{*} \frac{\partial}{\partial z^{*}} \text {. }
$$

c) $\alpha=0, A_{2}=a_{0}^{2},\left(a_{0}^{2} \in \mathbf{C}^{*}\right)$ et il existe un polynôme holomorphe $h$ nul à l'origine, tel que le changement de variables $\left(w^{*}, z^{*}\right)=\left(w+h(z), \frac{1}{a_{0}^{2}} z\right)$ transforme $M$ en $M^{*}=\left\{I m w^{*}+\sum_{k=1}^{N} \alpha_{k}\left(\operatorname{Im} z^{*}\right)^{2 k}=0\right\}$ et $\vec{X}$ en $\frac{\partial}{\partial z^{*}}$.

noindent Si $\vec{X}$ est la forme (iii) où $\alpha \in \mathbb{R}$ et $A_{j}, B_{j}$ sont des polynômes tels que $A_{2} \neq \equiv$ et $\operatorname{deg}\left(B_{2}\right) \leq \operatorname{deg}(P)$, alors $\alpha \neq 0, A_{2}=a_{0}^{2}+a_{1}^{2} z,\left(a_{0} \in\right.$ C, $a_{1} \in \mathbf{C}^{*}$ ) et il existe un polynôme holomorphe $h$ tel que le changement de variables $\left(w^{*}, z^{*}\right)=\left(w+h(z), a_{0}^{2}+a_{1}^{2} z\right)$

transforme $M$ en $M^{*}=\left\{I m w^{*}+C\left|z^{*}\right|^{2 m}=0\right\}$,

$C \in \mathbb{R}^{*}$ et $m \in \mathbb{N}^{*}$.

La dernière partie de cet article est consacréc aux applications holomorphes propres entre domaines polynomiaux rigides de $\mathbf{C}^{2}$. Ces applications étant ramifiées, il est crucial d'extraire le plus d'informations possible d'une ćtude locale. Pour cela, nous considérons les images directes du champ $\frac{\partial}{\partial w}$. Nous commençons par montrer que ces champs sont nécessairement polynômiaux puis nous utilisons les résultats précédents. Cela nous conduit au: 
Théorème 2 : Soient $P_{1}$ et $P_{2}$ deux polynômes sous-harmoniques sans termes harmoniques et $\Omega p_{j}(j=1,2)$ les domaines de $\mathbf{C}^{2}$ définis par

$\Omega p_{j}=\{\operatorname{Im} w+P(z, \bar{z})<0\}$.

noindent Soit $f: \Omega p_{1} \longrightarrow \Omega p_{2}$

une application holomorphe propre. Si $b \Omega p_{1}$ est strictement pseudoconvexe et non sphérique en au moins un point alors: $f=\left(\alpha w+f_{1}(z), f_{2}(z)\right)$ où $\alpha \in \mathbb{R}^{*}$

et $f_{1}, f_{2}$ sont des polynômes.

En ce qui concerne les biholomorphismes de domaines polynômiaux rigides de $\mathbf{C}^{2}, K$. Oeljeklaus ([4]) avait obtenu une classification et, en articulier, un analogue du théorème 2 sans hypothèse de non sphéricité. Cependant sa méthode ne s'adapte pas au cas ramifié traité ici. si l'on caractérisait les hypersurfaces polynômiales rigides et sphériques en tout point de stricte pseudoconvexité (nous conjecturons qu'elles sont de la forme $M=:\left\{\operatorname{Imw}+|p(z)|^{2}=0\right\}$ où $p$ est un polynôme holomorphe) alors notre résultat généraliserait complétement celui de ([4]) . Le problème de l'équivalence holomorphe entre domaines polynomiaux rigides de $\mathbf{C}^{n+1}$ n'est à ce jour pas résolu, les seuls résultats connus sont dus à $B$. Coupet et $S$. Pinchuk et concernent le cas homogène pondéré ([3]).

\section{Notations :}

- $\operatorname{deg}(P)$ désigne le degré d'un polynôme $P$ sur $\mathbf{C}$.

- $\mathcal{P}$ désigne le cône des polynômes sous-harmoniques sans termes harmoniques sur $\mathbf{C}$.

- $\mathcal{H}$ désigne l'algèbre des polynômes holomorphes sur $\mathbf{C}$.

Ce travail a été en partic réalisé lors de mon séjour à l'Université de Lille, je remercie cette institution pour son hospitalité et $F$. Berteloot pour de fructueuses discussions.

\section{Calcul explicite de l'algèbre de Lie $\mathcal{L}(M)$}

On établit ici le théorème 1 . Nous commençons par montrer que $\vec{X}$ est de la forme (i), (ii) ou (iii) où les $A_{j}, B_{j}$ sont des fonctions holomorphes au voisinage de l'origine. 
Nous utilisons pour cela le lemme suivant, dont la preuve sera donnée à la fin de cette partie.

Lemme 1 : Soient $P \in \mathcal{P}$

tel que $\triangle P(0,0) \neq 0, F_{1}(z), G_{1}(z), F_{2}(z)$ et $G_{2}(z)$ des fonctions holomorphes au voisinage de l'origine de $\mathbf{C}$.

i) Si $\operatorname{Re}\left[\left(F_{1}+F_{2} \frac{\partial P}{\partial z}\right) e^{i \alpha P}\right] \equiv 0$ et $\alpha \in \mathbb{R}^{*}$, alors $F_{1} \equiv F_{2} \equiv 0$

ii) Si $\left(F_{1}+F_{2} \frac{\partial P}{\partial z}\right)+\left(\bar{G}_{1}+\bar{G}_{2} \frac{\partial P}{\partial \bar{z}}\right) e^{i \alpha P} \equiv 0$ et $\alpha \neq 0$, alors $F_{1} \equiv F_{2} \equiv$ $G_{1} \equiv G_{2} \equiv 0$.

Conme $M$ n'est pas sphérique à l'origine, l'algèbre de Lie $\mathcal{L}(M)$ des champs de vecteurs holomorphes tangents à $M$ est de dimension réelle au plus égale à 3 et donc la famille $\left\{\frac{\partial}{\partial w}, \vec{X},\left[\vec{X}, \frac{\partial}{\partial w}\right],\left[\left[\vec{X}, \frac{\partial}{\partial w}\right], \frac{\partial}{\partial w}\right]\right\}$ n'est pas libre. Nous distinguerons trois cas.

$$
\mathbf{1}^{\text {er }} \text { cas : }\left\{\frac{\partial}{\partial w}, X\right\} \text { n'est pas libre }
$$

Dans ce cas $\vec{X}$ est de la forme (i).

$2^{\grave{e ̀ m e}}$ cas : $\left\{\frac{\partial}{\partial w}, \vec{X}\right\}$ est libre et $\left\{\frac{\partial}{\partial w}, \vec{X},\left[\vec{X}, \frac{\partial}{\partial w}\right]\right\}$ ne l'est pas. Le champ $\vec{X}=X_{1} \frac{\partial}{\partial w}+X_{2} \frac{\partial}{\partial z}$ satisfait l'équation différentielle:

$$
\frac{\partial X_{1}}{\partial w}+\alpha_{1} X_{1}+\alpha_{0}=0 \text { et } \frac{\partial X_{2}}{\partial w}+\alpha_{1} X_{2}=0
$$

où: $\alpha_{1}, \alpha_{0} \in \mathbb{R}$.

Intégrons l'équation (1). Si $\alpha_{1}$ était non nul on aurait:

$$
\vec{X}=\left(A_{1}(z) e^{-\alpha_{1} w}-\frac{\alpha_{0}}{\alpha_{1}}\right) \frac{\partial}{\partial w}+A_{2}(z) e^{-\alpha_{1} w} \frac{\partial}{\partial z}
$$

d'où, $\vec{X}$ étant tangent à $M$ :

$$
\operatorname{Re}\left[\left(\frac{A_{1}}{2 i}+A_{2} \frac{\partial P}{\partial z}\right) e^{i \alpha_{1} P}\right] \equiv 0
$$


D'après le lemme 1. i), on aurait alors $A_{1} \equiv A_{2} \equiv 0$ et donc $\vec{X}=$ $-\frac{\alpha_{0}}{\alpha_{1}} \frac{\partial}{\partial w}$ ce qui est exclu. Ainsi $\alpha_{1}=0$ et $\vec{X}$ est de la forme (ii). Comme $\vec{X}$ est tangent à $M, A_{2}$ ne peut être identiquement nul car on aurait alors $\alpha P-\operatorname{Im} A_{1} \equiv 0$ et donc $\vec{X}=A_{1}(0) \frac{\partial}{\partial w}$ avec $A_{1}=A_{1}(0) \in \mathbb{R}$.

$\mathbf{1}^{\text {ème }}$ cas : $\left\{\frac{\partial}{\partial w}, \vec{X},\left[\vec{X}, \frac{\partial}{\partial w}\right]\right\}$ est libre.

Le champ $\vec{X}$ satisfait l'équation différentielle:

(2.4) $\frac{\partial^{2} X_{1}}{\partial w^{2}}+\alpha_{2} \frac{\partial X_{1}}{\partial w}+\alpha_{1} X_{1}+\alpha_{0} \equiv 0$ et $\frac{\partial^{2} X_{2}}{\partial w^{2}}+\alpha_{2} \frac{\partial X_{2}}{\partial w}+\alpha_{1} X_{2} \equiv 0$ où $\alpha_{0}, \alpha_{1}, \alpha_{2} \in \mathbb{R}$.

Intégrons l'équation (4). Si $\alpha_{1}^{2}+\alpha_{2}^{2}$ était non nul, alors $\vec{X}$ serait de l'une des deux formes suivantes:

$$
\vec{X}=\left(\left(A_{1}(z) w+B_{1}(z)\right) e^{r w}+a\right) \frac{\partial}{\partial w}+\left(\left(A_{2}(z) w+B_{2}(z)\right) e^{r w} \frac{\partial}{\partial z}\right.
$$

où $a \in \mathbb{R}$ et $r \in \mathbb{R}^{*}$.

$$
\begin{array}{r}
\vec{X}=\left(A_{1}(z) e^{r_{1} w}+B_{1}(z) e^{r_{2} w}+a\right) \frac{\partial}{\partial w}+ \\
+\left(A_{2}(z) e^{r_{1} w}+B_{2}(z) e^{r_{2} w}\right) \frac{\partial}{\partial z}
\end{array}
$$

où $a \in \mathbb{R}, r_{1} \neq r_{2} \in \mathscr{C}$ et $r_{1}=\bar{r}_{2}$ si $r_{1} \notin \mathbb{R}$.

Supposons que $\vec{X}$ soit de la forme (5), $\vec{X}$ étant tangent à $M$ on aurait:

$$
\begin{array}{r}
\operatorname{Re}\left[\left(\frac{A_{1}}{2 i}+A_{2} \frac{\partial P}{\partial z}\right) e^{-i r P}\right] \equiv 0 \text { et } \operatorname{Re}\left[\left(B_{1}+B_{2} \frac{\partial P}{\partial z}\right) e^{-i r P}\right] \equiv \\
\equiv P \operatorname{Re}\left[\left(\frac{A_{1}}{2}+i A_{2} \frac{\partial P}{\partial z}\right) e^{-i r P}\right]
\end{array}
$$

et donc $\vec{X}=a \frac{\partial}{\partial w}$ d'après le lemme 1 . i) ce qui est exclu.

Supposons que $\vec{X}$ soit de la forme (6), comme $\left[\left[\vec{X}, \frac{\partial}{\partial w}\right], \frac{\partial}{\partial w}\right]$ est tangent à $M$, on aurait:

$$
\begin{aligned}
& R e\left\{r_{1}^{2}\left[e^{r_{1}(t-i P)}\left(\frac{A_{1}}{2 i}+A_{2} \frac{\partial P}{\partial z}\right)\right]+\right. \\
& \left.+r_{2}^{2}\left[e^{r_{2}(t-i P)}\left(\frac{B_{1}}{2 i}+B_{2} \frac{\partial P}{\partial z}\right)\right]\right\} \equiv 0
\end{aligned}
$$


pour tout $t \in \mathbb{R}$.

Dans le cas où $r_{1}$ et $r_{2}$ sont réels, on déduit immédiatement de (8) et du lemme 1. i) que $\vec{X}=a \frac{\partial}{\partial w}$. Ceci est exclu.

Dans le cas où $r_{1}$ et $r_{2}$ sont conjugués on peut écrire (8) sous la forme:

$$
\forall t \in \mathbb{R}: \operatorname{Re}\left(e^{i \beta t} r_{1}^{2} \widehat{A}+e^{-i \beta t} r_{2}^{2} \widehat{B}\right) \equiv 0
$$

où $\beta=I m r_{1}=I m r_{2} \neq 0, \widehat{A}=e^{-i r_{1} P}\left(\frac{A_{1}}{2 i}+A_{2} \frac{\partial P}{\partial z}\right)$ et $\widehat{B}=$ $e^{-i r_{2} P}\left(\frac{B_{1}}{2 i}+B_{2} \frac{\partial P}{\partial z}\right)$.

De (9) on tire:

$$
(\widehat{A}+\bar{B}) \equiv 0
$$

ou encore:

$$
\left(\frac{A_{1}}{2 i}+A_{2} \frac{\partial P}{\partial z}\right)+e^{2 i r_{1} P}\left(-\frac{\bar{B}_{1}}{2 i}+\bar{B}_{2} \frac{\partial P}{\partial \bar{z}}\right) \equiv 0
$$

Ce qui est exclu par le lemme 1. ii).

Ainsi $\alpha_{1}=\alpha_{2}=0$ et $\vec{X}$ est de la forme (iii), de plus comme $\left[\vec{X}, \frac{\partial}{\partial w}\right]$ est tangent à $M$, on voit que $A_{2}$ ne peut être identiquement nul car on aurait alors $\left[\vec{X}, \frac{\partial}{\partial w}\right]=A_{1}(0) \frac{\partial}{\partial w}$ avec $A_{1}=A_{1}(0) \in \mathbb{R}$.

Nous déterminons maintenant la forme exacte des fonctions $A_{j}$ et $B_{j}$, on peut, sans perte de généralité, supposer que $\triangle P(0,0)=1$. Ecrivons alors $P$ sous la forme

$$
P=|z|^{2}+2|z|^{2} \operatorname{Re} h(z)+2 \sum_{k=2}^{N}|z|^{2 k} \operatorname{Re} H_{k}(z)
$$

où $h(z)=z(1+q(z))$ et les $H_{k}(z)$ sont des polynômes holoporphes, $q(0)=0$.

Effectuons le changement de variable $G(w, z)=\left(w^{*}, z^{*}\right)=(w, g(z))$ où $g(z)=z(1+h(z))$. Comme le montre un calcul élémentaire, l'hypersurface $M^{*}=G(M)$ est défini au voisinage de l'origine par:

$$
\begin{array}{r}
I m w^{*}+\left|z^{*}\right|^{2}+2 \sum_{k \geq 2}\left|z^{*}\right|^{2 k} \operatorname{ReH}_{k}^{*}\left(z^{*}\right)= \\
=I m w^{*}+\left|z^{*}\right|^{2}+\lambda^{*}(z) \equiv 0
\end{array}
$$


où les $H_{k}^{*}$ sont des fonctions holoporphes et en particulier:

$$
H_{2}=\frac{1}{2}+q+\overline{H_{2}^{*}}(0)\left(2 h+h^{2}\right)+\left(H_{2}^{*} \circ g\right)(1+h)^{2}
$$

Soit $\vec{X}$ un champ de vecteurs holomorphe tangent à $M$ de la forme (iii). Le champ $\vec{X}^{*}=: G_{*} \vec{X}$ est de la forme:

$$
(i i i)^{*} \quad \vec{X}^{*}=\left(\alpha w^{* 2}+A_{1}^{*}\left(z^{*}\right) w^{*}+B_{1}^{*}\left(z^{*}\right)\right) \frac{\partial}{\partial w^{*}}+\left(A_{2}^{*}\left(z^{*}\right) w^{*}+B_{2}^{*}\left(z^{*}\right)\right) \frac{\partial}{\partial z^{*}}
$$

et les relations suivantes sont satisfaites:

$$
\begin{cases}A_{1}^{*} \circ g=A_{1}, & A_{2}^{*} \circ g=\frac{d g}{d z} A_{2} \\ B_{1}^{*} \circ g=B_{1} & B_{2}^{*} \circ g=\frac{d g}{d z} B_{2}\end{cases}
$$

En exprimant que les champs $\left[\overrightarrow{X^{*}}, \frac{\partial}{\partial w^{*}}\right]$ puis $\overrightarrow{X^{*}}$ sont tangents à $M^{*}$ on obtient les équations:

$$
\begin{array}{r}
\operatorname{Re}\left[\frac{A_{1}^{*}}{2 i}+A_{2}^{*}\left(\overline{z^{*}}+\frac{\partial \lambda^{\star}}{\partial z^{*}}\right)\right]=\alpha\left(\left|z^{*}\right|^{2}+\lambda^{*}\right) \\
\operatorname{Re}\left[\frac{B_{1}^{*}}{2 i}+B_{2}^{*}\left(\overline{z^{*}}+\frac{\partial \lambda^{\star}}{\partial z^{*}}\right)\right]= \\
=\left(\left|z^{*}\right|^{2}+\lambda^{*}\right) \operatorname{Re}\left[\frac{A_{1}^{*}}{2}+i A_{2}^{*}\left(\overline{z^{*}}+\frac{\partial \lambda^{*}}{\partial z^{*}}\right)\right]
\end{array}
$$

Nous allons utiliser ces équations pour déterminer les fonctions holomorphes $A_{j}^{*}$ et $B_{j}^{*}$, introduisons à cet effet les notations suivantes:

$$
\begin{aligned}
A_{j}^{*} & =a_{0}^{* j}+a_{1}^{* j} z^{*}+z^{* 2} \widetilde{A}_{j}^{*} \\
B_{j}^{*} & =b_{0}^{* j}+b_{1}^{* j} z^{*}+z^{* 2} \widetilde{B}_{j}^{*} \\
H_{2}^{*} & =h_{0}^{* 2}+h_{1}^{* 2} z^{*}+z^{* 2} \widetilde{H}_{2}^{*}
\end{aligned}
$$

Dans la suite, $O\left(z^{*}, \overline{z^{*}}\right)$ désigne une fonction analytique réelle au voisinage de l'origine dont l'expression est susceptiple de varier au cours des calculs. Comme:

$$
\overline{z^{*}}+\frac{\partial \lambda^{*}}{\partial z^{*}}=\bar{z}^{*}+2\left|z^{*}\right|^{2}\left(\overline{z^{*}} h_{0}^{* 2}+\overline{z^{*} H_{2}^{*}}\right)+\left|z^{*}\right|^{4} O\left(z^{*}, \overline{z^{*}}\right),
$$


on peut écrire les équations (16) et (17) sous la forme:

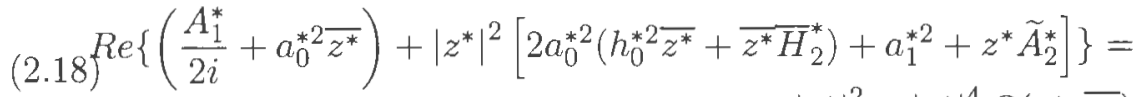

$$
=\alpha\left|z^{*}\right|^{2}+\left|z^{*}\right|^{4} O\left(z^{*}, \overline{z^{*}}\right)
$$

$$
\begin{array}{r}
\operatorname{Re}\left\{\left(\frac{B_{1}^{*}}{2 i}+b_{0}^{* 2} \overline{z^{*}}\right)+\left|z^{*}\right|^{2}\left[2 b_{0}^{* 2}\left(h_{0}^{* 2} \overline{z^{*}}+\overline{z^{*} \bar{H}_{2}^{*}}\right)+b_{1}^{* 2}+z^{*} \widetilde{B}_{2}^{*}\right]\right\}= \\
=\left(\frac{1}{2} \operatorname{Re}_{0}^{* 1}\right)\left|z^{*}\right|^{2}+\left|z^{*}\right|^{4} O\left(z^{*}, \overline{z^{*}}\right)
\end{array}
$$

On déduit alors immédiatement de (18) et (19) que:

$$
\begin{cases}A_{1}^{*}=a_{0}^{* 1}+a_{1}^{* 1} z, & \widetilde{A}_{2}^{*}=-2 \bar{a}_{0}^{* 2}\left(\bar{h}_{0}^{* 2}+H_{2}^{*}\right) \\ B_{1}^{*}=b_{0}^{* 1}+b_{1}^{* 1} z & B_{2}^{*}=-2 \bar{b}_{0}^{* 2}\left(\bar{h}_{0}^{* 2}+H_{2}^{*}\right)\end{cases}
$$

Il résulte de (14), (15) ct (20) que les fonctions $A_{j}$ et $B_{j}$ sont des fractions rationnelles qu'il est possible d'expliciter. En particulier, on vérifie que le degré de $B_{2}$ est inférieur à celui de $P$.

Lorsque $\vec{X}$ est de la forme (ii), on procède de façons identique à ceci près que seule l'équation (16) est à considérer.

Il s'avère que le polynôme $P$ est d'un type bien particulier lorsque les fonctions $A_{j}$ et $B_{j}$ sont des polynômes. Ceci fait l'objet de la propostion 1 suivante et jouera un rôle crucial dans la preuve du théorème 2 .

\section{Preuve de la proposition 1:}

Nous utiliserons le lemme suivant dont la démonstration est repoussée à la fin de cette partie.

Lemme 2 : Soient $a \in \mathbb{C}^{*}, \alpha \in I R^{*}, k \in \mathbb{N}, Q \in \mathcal{P}$ et $h \in \mathcal{H}$

i) Si $Q$ est homogène et $\operatorname{Re}\left(a z^{k} \frac{\partial Q}{\partial z}\right) \equiv 0$ alors $Q \equiv 0$ ou bien $k=$ $1, a \in i \mathbb{R}$ et $Q=C|z|^{2 m}\left(C \in \mathbb{R}^{*}, m \in \mathbb{N}^{*}\right)$

ii) Si $\operatorname{Re}\left(a z \frac{\partial Q}{\partial z}+h\right) \equiv \alpha Q$ alors $h \in i \mathbb{R}$ et $Q$ est homogène. De plus, $Q=C|z|^{2 m}$ lorsque Ima $\neq 0$. 
Supposons d'abord que $\vec{X}$ soit de la forme (i). Comme $\vec{X}$ est tangent à $M$ on a:

$$
\forall z \in \mathbb{C}: \operatorname{Re}\left[\frac{A_{1}(z)}{2 i}+A_{2}(z) \frac{\partial P}{\partial z}(z, \bar{z})\right]=\frac{\alpha}{2} P(z, \bar{z})
$$

Ecrivons $A_{2}$ sous la forme $A_{2}(z)=a_{0}^{2}+\ldots+a_{N}^{2} z^{N}$ où $a_{N}^{2} \neq 0$ et désignons par $H$ la partie homogène de plus haut degré de $P$.

Si $N$ était strictement supérieur à 1 alors d'aprés (21), on aurait $\operatorname{Re}\left(a_{N}^{2} z^{N} \frac{\partial H}{\partial z}\right) \equiv 0$. Mais ceci est impossible en vertu du lemme 2. i) et donc $N \leq 1$.

a) Si $\alpha \neq 0$ alors $\operatorname{Re}\left(a_{1}^{2} z \frac{\partial H}{\partial z}\right)=\frac{\alpha}{2} H$ et donc $N=1$. Soient alors $Q \in \mathcal{P}$ et $h \in \mathcal{H}$ tels que $P(z, \bar{z})=Q\left(a_{0}^{2}+a_{1}^{2} z, \bar{a}_{0}^{2}+\bar{a}_{1}^{2} \bar{z}\right)+2 \operatorname{Imh}\left(a_{0}^{2}+a_{1}^{2} z\right)$.

Le changement de variable: $\left(w^{*}, z^{*}\right)=\left(w+2 h\left(a_{0}^{2}+a_{1}^{2} z\right), a_{0}^{2}+a_{1}^{2} z\right)$ transforme $M$ en $M^{*}=\left\{I m w^{*}+Q\left(z^{*}, \overline{z^{*}}\right)=0\right\}$ et l'équation (21) devient:

$$
\forall z^{*} \in \mathbb{C}: \operatorname{Re}\left[\frac{A_{1}^{*}\left(z^{*}\right)}{2 i}+a_{1}^{2} z^{*} \frac{\partial Q}{\partial z^{*}}\left(z^{*}, \overline{z^{*}}\right)\right]=\frac{\alpha}{2} Q\left(z^{*}, \overline{z^{*}}\right)
$$

pour un certain $A_{1}^{*} \in \mathcal{H}$. Comme $\alpha \neq 0$, on déduit de (22) et du lemme 2.ii) que $Q$ est homogène et égal à $C|z|^{2 m}\left(C>0, m \in \mathbb{N}^{*}\right)$ lorsque $\operatorname{Im} a_{1}^{2} \neq 0$.

b) Si $\alpha=0$ et $a_{1}^{2} \neq 0$ alors $\operatorname{Re}\left(a_{1}^{2} z \frac{\partial H}{\partial z}\right) \equiv 0$ et donc $a_{1}^{2} \in i \mathbb{R}$ en vertu du lemme 2. i).

Effectuons le même changement de variable $F(w, z)=\left(w^{*}, z^{*}\right)$ que cidessus et observons que $F_{*} \vec{X}=A_{1}^{*}\left(z^{*}\right) \frac{\partial}{\partial w^{*}}+i \lambda_{2} z^{*} \frac{\partial}{\partial z^{*}}$ où $A_{1}^{*} \in \mathcal{H}$ et $\lambda_{2} \in \mathbb{R}^{*}$.

Ecrivons $Q$ sous la forme $Q=\sum_{k=1}^{N} 2\left|z^{*}\right|^{2 k} \operatorname{Re} H_{k}^{*}\left(z^{*}\right)$ où les $H_{k}^{*} \in \mathcal{H}$ et reportons dans l'equation (22). Comme $a_{1}^{2} \in i \mathbb{R}$, on voit facilement que les $H_{k}^{*}$ sont des constantes puis que $F_{*} \vec{X}$ prend la forme annoncée.

c) Si $\alpha=0$ et $a_{1}^{2}=0$. Soit $h \in \mathcal{H}$ tel que $h(0)=0$ et $\frac{d h}{d z}=-\frac{1}{a_{0}^{2}} A_{1}(z)$. Le changement de variables $\left(w^{*}, z^{*}\right)=\left(w+h(z), \frac{1}{a_{0}^{2}} z\right)$ transforme $\vec{X}$ en $\frac{\partial}{\partial z^{*}}$ 
et $M$ en $M^{*}=\left\{I m w^{*}+Q\left(z^{*}, \overline{z^{*}}\right)=0\right\}$ où $Q$ est un polynôme réel pourant contenir des termes harmoniques. On voit immédiatement en traduisant le fait que $\frac{\partial}{\partial z^{*}}$ est tangent à $M^{*}$ que $Q$ ne dépend que de $\operatorname{Im} z^{*}$.

Considérons maintenant le cas où $\vec{X}$ est de la forme (ii). En traduisant le fait que $\vec{X}$ et $\left[\frac{\partial}{\partial w}, \vec{X}\right]$ sont tangents à $M$ on obtient les équations:

$$
\forall z \in \mathbb{C}: \operatorname{Re}\left[\frac{A_{1}(z)}{2 i}+A_{2}(z) \frac{\partial P}{\partial z}(z, \bar{z})\right]=\alpha P(z, \bar{z})
$$

et

$\forall z \in \mathbb{C}: \operatorname{Re}\left[\frac{B_{1}(z)}{2 i}+B_{2}(z) \frac{\partial P}{\partial z}(z, \bar{z})\right]=P(z, \bar{z}) \operatorname{Re}\left[\frac{A_{1}(z)}{2}+i A_{2}(z) \frac{\partial P}{\partial z}(z, \bar{z})\right]$

En procédant comme avec l'équation (21), on déduit de (23) que $A_{2}(z)=$ $a_{0}^{2}+a_{1}^{2} z$ où $a_{0}^{2}, a_{1}^{2} \in \mathbb{C}$. Nous allons montrer que $\alpha \neq 0$. Pour cela, procédons par l'absurde et supposons que $\alpha=0$. Soit $H$ la partie homogène de plus haut degré de $P$. De (23) on déduit que $\left.R e\left(a_{1}^{2} z \frac{\partial H}{\partial z}\right)\right) \equiv 0$, de (24) et puisque $\operatorname{deg} B_{2} \leq \operatorname{deg} P$, on déduit que $\operatorname{Re}\left(i a_{1}^{2} z \frac{\partial H}{\partial z}\right) \equiv 0$. Il resulte alors du lemme 2.i) que $a_{1}^{2}=0$. Ainsi, $\vec{X}=\left(A_{1}(z) w+B_{1}(z)\right) \frac{\partial}{\partial w}+\left(a_{0}^{2} w+\right.$ $\left.B_{2}(z)\right) \frac{\partial}{\partial z}$ et $\vec{Y}=\left[\frac{\partial}{\partial w}, \vec{X}\right]=A_{1}(z) \frac{\partial}{\partial w}+a_{0}^{2} \frac{\partial}{\partial z}$. soit $h \in \mathcal{H}$ tel que $h(0)=0$ et $\frac{d h}{d z}=-\frac{1}{a_{0}^{2}} A_{1}(z)$. Comme dans l'étude du cas c), le changement de variables $F(z, w)=\left(w^{*}, z^{*}\right)=\left(w+h(z), \frac{1}{a_{0}^{2}} z\right)$ transforme $M \mathrm{cn}$ $M^{*}=\left\{I m w^{*}+f\left(I m z^{*}\right)=0\right\}$ où $f$ est un polynôme réel nul en 0 . De plus, $F_{*} \vec{X}=\vec{X}^{*}=B_{1}^{*}\left(z^{*}\right) \frac{\partial}{\partial w^{*}}+\left(w^{*}+B_{2}^{*}\left(z^{*}\right)\right) \frac{\partial}{\partial z^{*}}$ et $F_{*} \vec{Y}=\frac{\partial}{\partial z^{*}}$ où $B_{1}^{*}, B_{2}^{*} \in \mathcal{H}$. Comme par hypothèse $M^{*}$ est non sphérique à l'origine, $\left[\frac{\partial}{\partial z^{*}}, \vec{X}^{*}\right]$ est une combinaison linéaire réelle de $\frac{\partial}{\partial w^{*}}, \vec{X}^{*}$ et $\frac{\partial}{\partial z^{*}}$. On a donc:

$$
\frac{d B_{1}^{*}}{d z^{*}} \frac{\partial}{\partial w^{*}}+\frac{d B_{2}^{*}}{d z^{*}} \frac{\partial}{\partial z^{*}}=\alpha_{1} \frac{\partial}{\partial w^{*}}+\alpha_{2} \vec{X}+\alpha_{3} \frac{\partial}{\partial z^{*}}
$$


d'où l'on déduit immédiatement que $\alpha_{2}=0$ et que $B_{1}^{*}, B_{2}^{*}$ sont de degré au plus égal à 1 . En traduisant le fait que $\vec{X}^{*}$ est tangent à $M$ on obtient:

$$
\operatorname{Re}\left[\frac{B_{1}^{*}\left(z^{*}\right)}{2 i}-\frac{1}{2} f\left(\operatorname{Im} z^{*}\right) f^{\prime}\left(\operatorname{Im} z^{*}\right)+\frac{B_{2}^{*}\left(z^{*}\right)}{2 i} f^{\prime}\left(\operatorname{Im} z^{*}\right)\right] \equiv 0
$$

Enfin, comme $B_{1}^{*}$ et $B_{2}^{*}$ sont de degrés au plus 1 , on déduit de (26) que $f$ est de degré 1 . Ceci est impossible puisque $M^{*}$ est non dégénérée en $(0,0)$.

On a donc $\alpha \neq 0$, ct comme dans l'étude du cas a), il résulte de (23) que $a_{1}^{2} \neq 0$ et que $M$ est transformćc cn une hypersurface $M^{*}=$ $\left\{I m w^{*}+H^{*}\left(z^{*}, \overline{z^{*}}\right)=0\right\}$ où $H^{*}$ est homogènes et équivalent à $P$. Comme deux polynomes équivalents ont mêmes parties honıgènes de plus haut degré, on a $H^{*}=H$. Or, comme nous l'avons déja observé, on peut déduire de (24) que $\operatorname{Re}\left(i a_{1}^{2} z \frac{\partial H}{\partial z}\right) \equiv 0$. Il resulte alors du lemme 2.i) que $H=M|z|^{2 m}$ et cola achève la démonstration.

\section{Preuve du lemme 2 :}

Pour la preuve de i) (cf:[2] lemme 2.3). Passons maintenant à la preuve de ii). Puisque $Q$ est sans termes harmoniques on voit que $h$ est une constante imaginaire pure.

posons $Q(z, \bar{z})=\sum_{p, q \geq 1} A_{p, q} z^{p} \bar{z}^{q} ;$ on a par hypothèse:

$$
\sum_{p, q \geq 1} A_{p q}[-2 \alpha+(p+q) \operatorname{Rea}+i(p-q) \operatorname{Im} a] z^{p} \bar{z}^{q} \equiv 0
$$

Comme $\alpha \neq 0$ par hypothèse, on obtient facilement de (27) que $Q$ est homogène et, si de plus $\operatorname{Im} a \neq 0$, alors $Q=C|z|^{2 m}, C>0$ et $m \in \mathbb{N}^{*}$.

\section{Preuve de lemme 1 :}

Il suffit de donner la preuve de ii). En effet: on a $2 e^{-i \alpha P} \operatorname{Re}\left[\left(F_{1}+F_{2} \frac{\partial P}{\partial z}\right) e^{i \alpha P}\right]=\left[\left(F_{1}+F_{2} \frac{\partial P}{\partial z}\right)+\left(\bar{F}_{1}+\bar{F}_{2} \frac{\partial P}{\partial \bar{z}}\right) e^{-2 i \alpha P}\right]$, on est donc ramené au cas ii). On peut sans perte de généralité, supposer que $\triangle P(0,0)=1$. Ecrivons alors $P$ sous la forme: $P(z, \bar{z})=|z|^{2}+\bar{z} A(z)+$ 
$z \overline{A(z)}+\sum_{p, q \geq 2} A_{p q} z^{p} \bar{z}^{q}$ où $A_{p q}=\bar{A}_{q p}$ et $A$ un polynôme tel que $A(0)=$ $A^{\prime}(0)=0$.

Notons:

$$
\begin{gathered}
T(z)=2 \sum_{p \geq 2} A_{p 2} z^{p} \\
F_{2}^{*}(z)=F_{2}(z)-F_{2}(0) \\
G_{2}^{*}(z)=G_{2}(z)-G_{2}(0)
\end{gathered}
$$

On a, par hypothèse:

$$
\left(F_{1}+F_{2} \frac{\partial P}{\partial z}\right)+\left(\bar{G}_{1}+\bar{G}_{2} \frac{\partial P}{\partial \bar{z}}\right) e^{i \alpha P} \equiv 0 \text { et } \alpha \neq 0 .
$$

Supposons que $F_{1}, F_{2}, G_{1}$, et $G_{2}$ sont non identiquement nulles, et montrons que cela conduit à contradiction. Montrons d'abord que $F_{1}$ et $G_{1}$ sont des polynômes et $F_{2}$ et $G_{2}$ sont des fractions rationnelles. En collectant les termes harmoniques on déduit de (28) que:

$$
F_{1}+F_{2}(0)(\bar{z}+\bar{A})+\bar{G}_{1}+\overline{G_{2}(0)}(z+A) \equiv 0
$$

et donc que $F_{1}$ et $G_{1}$ sont des polynômes.

En identifiant maintenent les termes de la forme $z^{p} \bar{z}$ ou $\bar{z}^{q} z ; p, q \geq 1$ dans (28), on obtient:

$$
\begin{aligned}
& \bar{z}\left\{\left(1+A^{\prime}\right) F_{2}^{*}+\overline{G_{2}(0)} T+i \alpha \overline{G_{1}(0)}(z+A)\right\}+ \\
& \quad+z\left\{F_{2}(0) \bar{T}+\left(1+\overline{A^{\prime}}\right) \overline{G_{2}^{*}}+i \alpha \overline{G_{1}(0)} \bar{A}\right\} \equiv 0
\end{aligned}
$$

ou encore:

$$
\begin{aligned}
& \frac{1}{z}\left\{\left(1+A^{\prime}\right) F_{2}^{*}+\overline{G_{2}(0)} T+i \alpha \overline{G_{1}(0)}(z+A)\right\}+ \\
& \left.+\frac{1}{\bar{z}}\left\{F_{2}(0) \bar{T}+\left(1+\overline{A^{\prime}}\right) \overline{G_{2}{ }^{*}}+i \alpha G_{1}(0) \bar{A}\right\} \equiv 0\right\}
\end{aligned}
$$

De (30) on voit que $F_{2}^{*}$ et $G_{2}^{*}$ sont des fractions rationnelles, et donc $F_{2}$ et $G_{2}$ le sont également. Ainsi l'égalité (28) peut s'écrire sous la forme:

$$
\frac{Q_{1}(z, \bar{z})}{Q_{2}(z, \bar{z})}=e^{i \alpha P(z, \bar{z})}
$$

où $Q_{1}, Q_{2}$ sont despolynômes en $z, \bar{z}$. 
Posons: $z=\sigma t$, pour un certain $\sigma$ fixé dans $C^{*}$ et $t$ varie dans un voisinage de l'origine dans $\mathbb{R}$, en remplaçant $z$ par $\sigma t$ dans (31) on obtient:

$$
\frac{Q_{1}(\sigma t, \bar{\sigma} t)}{Q_{2}(\sigma t, \bar{\sigma} t)}=e^{i \alpha P(\sigma t, \bar{\sigma} t)}
$$

En dérivant (32) par raport à $t$, on obtient:

$$
Q_{1}^{\prime} Q_{2}-Q_{1} Q_{2}^{\prime}=i \alpha P^{\prime} Q_{1} Q_{2}
$$

En comparant les degrés des deux membres de l'égalité (33), et puisque $\alpha \neq 0$, on obtient donc une contradiction.

\section{Description des applications holomorphes propres entre domaines polynômiaux rigides}

Nous établissons ici le théorème 2, pour cela nous utiliserons la proposition suivante qui est en fait une amélioration du théorème 2 de [2].

Propositon 2 : Soient $\Omega_{1}$ et $\Omega_{2}$ deux domaines de la forme

$$
\Omega_{j}:\left\{(w, z) \in \mathbb{C}^{2}: R e w+P_{j}(z, \bar{z})<0\right\}
$$

où $P_{j} \in \mathcal{P}$. Soit $f: \Omega_{1} \longrightarrow \Omega_{2}$ une application holomorphe telle que:

1) $f=\left(f_{1}, f_{2}\right)$ se prolonge en un biholomorphisnc local d'un voisinage $U_{1}$ de l'origine sur un autre noté $U_{2}$.

2) $f_{2}(w, 0) \equiv 0$ et $f_{1}(0,0)=0$.

Alors on se trouve dans l'un des deux cas suivants:

i) $f_{1}(w, z)=\Gamma w ;(\Gamma>0)$ et $f_{2}(w, z)=f_{2}(z)$ où $f_{2}$ est un polynôme.

ii) $f_{1}(w, z)=\frac{\Gamma w}{1+i \lambda w} ;\left(\Gamma>0, \lambda \in \mathbb{R}^{*}\right)$ et $f_{2}(w, z)=\frac{g(z)}{(1+i \lambda w)^{1 / m}}$; $P_{2}(z, \bar{z})=C|z|^{2 m}$ et $P_{1}(z, \bar{z})=\frac{C}{\Gamma}|g(z)|^{2 m}$ avec $C>0$ et $m \in \mathbb{N}^{*}$ et $g$ est un polynồme. 


\section{Preuve du théorème 2 :}

Nouss prodédons en plusieurs étapes.

$\mathbf{1}^{\text {ème }}$ étape: On se ramène au cas où $f$ induit un biholoporphisme local au voisinage de $\zeta_{0}=(0,0), f\left(\zeta_{0}\right)=\zeta_{0}$ et $\mathrm{b} \Omega_{P_{1}}, b \Omega_{P_{2}}$ sont strictement pseudoconvexes non sphériques en $\zeta_{0}$.

En argumentant comme dans la preuve du théorème 2 de $([2])$, on établit l'existence d'une suite $\left(w_{n}, z_{n}\right)$ dans $\Omega_{P_{1}}$ qui converge vers $\zeta_{0} \in b \Omega_{P_{1}}$ et telle que $f\left(w_{n}, z_{n}\right)$ converge vers un point de stricte pseudo-convexité de $b \Omega_{P_{2}}$. Alors, d'après ([1]), $f$ se prolonge continuement à $b \Omega_{P_{1}}$ au voisinage de $\zeta_{0}$. En remplaçant $\zeta_{0}$ par un point arbitrairement voisin, on peut supposer que $b \Omega_{P_{1}}$ soit strictement pseudoconvexe en $\zeta_{0}$. Dans ces conditions $f$ induit un difféomorphisme en $\zeta_{0}$ et se prolonge holomorphiquement au voisinage de ce point par le principe de réflexion de Pinchuk. On se ramène à $\zeta_{0}=(0,0)$ en composant $f$ avec des automorphismes adéquats de $\mathbb{C}^{2}$.

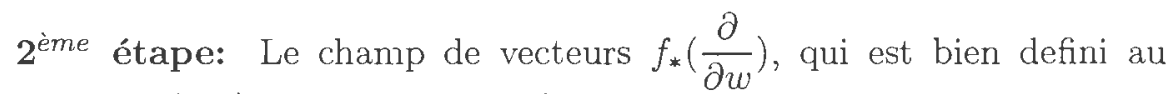
voisinage de $(0,0)$, est de l'une des formes suivantes:

(1) $\left(\alpha w^{2}+A_{1}(z) w+B_{1}(z)\right) \frac{\partial}{\partial w}+\left(A_{2}(z) w+B_{2}(z)\right) \frac{\partial}{\partial z}$

(2) $\left(\alpha w+A_{1}(z)\right) \frac{\partial}{\partial w}+A_{2}(z) \frac{\partial}{\partial z}$

(3) $\lambda \frac{\partial}{\partial w}$

où $\alpha \in \mathbb{R}, \lambda \in \mathbb{R}^{*}$ et $A_{j}, B_{j}$ sont de polynômes tels que $A_{2} \neq 0$ et $\operatorname{deg} B_{2} \leq \operatorname{deg} P_{2}$.

Compte tenu de la première étape et du théorème 1 , on sait que $f_{*}\left(\frac{\partial}{\partial w}\right)$ est de l'une de ces formes à ceci près que $A_{2}$ et $B_{2}$ sont remplacés par $\frac{\widetilde{A}_{2}}{1+H_{2}}$ et $\frac{\widetilde{B}_{2}}{1+H_{2}}$ où $\widetilde{A}_{2}, \widetilde{B}_{2}$ et $H_{2}$ sont des polynônıes holomorphes tels que $\widetilde{A}_{2} \neq 0$ et $\operatorname{deg} \widetilde{B}_{2} \leq \operatorname{deg} P+\operatorname{deg} H_{2}$. En utilisant le fait que $f$ est holomorphe propre, nous allons montrer que les fractions $\frac{\widetilde{A}_{2}}{1+H_{2}}$ et $\frac{\widetilde{B}_{2}}{1+H_{2}}$ sont en fait 
des polynômes. Nous étudions le cas d'un champ de la forme (1), l'autre se traitant de façon parfaitement identique.

Soicnt $U$ un voisinage de l'origine sur le quel $f_{*}\left(\frac{\partial}{\partial w}\right)$ est defini et $\left(w_{0}, z_{0}\right)$ un point fixé dans $U \backslash f\left(V_{f}\right)$.

Supposons que l'une des fractions $\frac{\widetilde{A}_{2}}{1+H_{2}}$ et $\frac{\widetilde{B}_{2}}{1+H_{2}}$ ait des pôles. Alors, on peut trouver $\left(w_{1}, z_{1}\right) \in \Omega_{P_{2}}$ tel que:

$$
\lim _{(w, z) \longrightarrow\left(w_{1}, z_{1}\right)}\left|\frac{\tilde{A}_{2}(z) w+\widetilde{B}_{2}(z)}{1+H_{2}(z)}\right|=+\infty
$$

Remarquons que $\frac{\widetilde{A}_{2}(z) w+\widetilde{B}_{2}(z)}{1+H_{2}(z)}$ est holomorphe sur $\Omega_{l^{\prime}} \backslash S$, où $S$ est un ensemble analytique de la forme $\left\{(w, z) \in \Omega_{P_{2}}: \Pi_{k=1}^{N}\left(z-z_{k}\right)=0\right\}$. Comme $f$ est holomorple propre, un théorème de Remmert stipule que $f\left(V_{f}\right)$ est un ensemble analytique de $\Omega_{P_{2}}$.

Puisque $\Omega_{P_{2}} \backslash f\left(V_{f}\right) \cup S$ est connexe et dense dans $\Omega_{P_{2}}$, il existe un chemin continu $\gamma:[0,1] \longrightarrow \Omega_{P_{2}}$ tel quc: $\gamma(0)=\left(w_{0}, z_{0}\right), \gamma(1)=\left(w_{1}, z_{1}\right)$ et $\gamma(t) \notin f\left(V_{f}\right) \cup S$ pour tout $t \in[0,1[$.

L'application $f$ induisant un revêtement fini de $\Omega_{P_{1}} \backslash f^{-1}\left[f\left(V_{f}\right)\right]$ sur $\Omega_{P_{2}} \backslash$ $V_{f}$, il existe un relévement $\widetilde{\gamma}$ de $\gamma$ sur $[0,1[$ par $f$, c'est à dire un chemin $\tilde{\gamma}:\left[0,1\left[\longrightarrow \Omega_{P_{1}} \backslash f^{-1}\left[f\left(V_{f}\right)\right]\right.\right.$ tel que $f o \tilde{\gamma}(t)=\gamma(t)$ pour tout $t \in[0,1[$.

Le champ $\vec{X}=f_{*}\left(\frac{\partial}{\partial w}\right)$ se prolonge le long de $\gamma([0,1[)$ en un champ $\overrightarrow{\widetilde{X}}$ tel que:

$$
\begin{array}{r}
\forall t \in\left[0,1\left[: \overrightarrow{\widetilde{X}}(\gamma(t))=\left[f_{*}\left(\frac{\partial}{\partial w}\right)\right]_{\gamma(t)}=\right.\right. \\
=\frac{\partial f_{1}}{\partial w}(\widetilde{\gamma}(t)) \frac{\partial}{\partial w}+\frac{\partial f_{2}}{\partial w}(\widetilde{\gamma}(t)) \frac{\partial}{\partial z}
\end{array}
$$

Par ailleurs, puisque $\vec{X}$ est donné par (1) sur $U$ et que $\gamma([0,1[) \subset$ $\Omega_{P_{2}} \backslash S$, le champ

$$
\left(\alpha w^{2}+A_{1}(z) w+B_{1}(z)\right) \frac{\partial}{\partial w}+\frac{A_{2}(z) w+B_{2}(z)}{1+H_{2}(z)} \frac{\partial}{\partial z}
$$

est également un prolongement de $\vec{X}$ le long de $\gamma([0,1[)$. D'aprés (35) et 
par unicité du prolongement on a donc:

$$
\forall t \in[0,1]: \frac{\partial f_{2}}{\partial w}(\tilde{\gamma}(t))=\frac{A_{2}\left(\gamma_{2}(t)\right) \gamma_{1}(t)+B_{2}\left(\gamma_{2}(t)\right)}{1+H_{2}\left(\gamma_{2}(t)\right)}
$$

Comme $f$ est propre et $f o \tilde{\gamma}=\gamma$ sur $[0,1[$ on voit que $\widetilde{\gamma}([0,1[)$ est relativement compact dans $\Omega_{P_{1}}$. Compte tenu de (34) il suffit alors de faire tendre $t$ vers 1 dans (36) pour obtenir une contradiction.

$3^{\text {ème }}$ étape $: f_{*}\left(\frac{\partial}{\partial w}\right)=\lambda \frac{\partial}{\partial w}$ et $f$ est de la forme annoncée.

Le polynôme $P_{2}$ ne peut être équivalent à un polynôme de la forme $|z|^{2 m}$ car $b \Omega_{P_{2}}$ n'est pas sphérique en tout point de stricte pseudoconvexité. Ainsi, la proposition 1 montre que, si $f_{*}\left(\frac{\partial}{\partial w}\right)$ est de la forme (1) ou (2), alors:

$$
f_{*}\left(\frac{\partial}{\partial w}\right)=\left(\alpha w+A_{1}(z)\right) \frac{\partial}{\partial w}+A_{2}(z) \frac{\partial}{\partial z}
$$

avec $A_{2}(z)=a_{0}^{2}+c z, \alpha \in \mathbb{R}^{*}$ et $c \in \mathbb{R}^{*}$.

ou, quitte à composer $f$ avec un automorphisme de $C^{2}$ de type $(w+$ $h(z), z+b)$ où $h \in \mathcal{H}, a \in \mathbb{C}^{*}$ et $b \in \mathbb{C}$, on se ramène au cas où $f_{*}\left(\frac{\partial}{\partial w}\right)$ est de l'une des formes suivantes:

$$
f_{*}\left(\frac{\partial}{\partial w}\right)=\lambda_{1} \frac{\partial}{\partial w}+i \lambda_{2} z \frac{\partial}{\partial z}
$$

où $\lambda_{1} \in \mathbb{R}$ et $\lambda_{2} \in \mathbb{R}^{*}$

ou bien

$$
f_{*}\left(\frac{\partial}{\partial w}\right)=\frac{\partial}{\partial z}
$$

Il est clair que seules les équations (37) et (38) sont à considérer. Nous allons montrer qu'aucune de ces deux équations ne peut être satisfaite. En intégrant l'équation (37) on obtient:

$$
f_{2}(w, z)=F_{2}(z) e^{c w}-F_{2}(0)
$$

où $F_{2}$ est une fonction entière.

En intégrant l'équation (38) on obtient:

$$
f_{2}(w, z)=F(z) e^{i \lambda_{2} w}
$$


où $F$ est une fonction entière et en composant $f$ avec un automorphisme de $C^{2}$ de type $(w+h(z), z+b)$ on se ramène au cas où $F(0)=0$.

De $(40)$ et $(41)$ on tire: $f_{2}(w, 0) \equiv 0$, compte tenu de la première étape et de la proposition 2, ceci est imposible puisque $\lambda_{2} \neq 0$ et $c \neq 0$.

Finalement, $f_{*}\left(\frac{\partial}{\partial w}\right)$ ne peut être que de la forme (3) ce qui après intégration donne:

$$
f(w, z)=\left(\lambda w+F_{1}(z), F_{2}(z)\right)
$$

où $F_{1}$ et $F_{2}$ sont entières nulles à l'origine, compte tenu de la première étape et de la propostion $2, f(w, z)=\left(\lambda w, F_{2}(z)\right)$ où $F_{2}$ est un polynôme. En composant $f$ avec un automorphisme de $\mathbb{C}^{2}$ de type $(w+h(z), z+b)$ où $h \in \mathcal{H}$ et $b \in \mathbb{C}$, on trouve alors la forme annoncée de $f$.

Nous terminons cette partie par la

\section{Preuve de la proposition 2 :}

Conmmençons par i). D'aprés théorène 2 de [2], (ici $\lambda=0), f_{1}(w, z)=$ $\Gamma w, \Gamma>0$, notons dans ce cas $f(w, z)=\left(\Gamma w, f_{2}(w, z)\right)$ et considérons le champ $f_{*}\left(i \frac{\partial}{\partial w}\right)$. C'est un champ de vecteurs holomorphes défini sur $U_{2}$ et tangent àb $\Omega_{2}$. D'aprés la preuve de la proposition 3.1 de [2], $f_{*}\left(i \frac{\partial}{\partial w}\right)$ est de la forme:

$$
\left[f_{*}\left(i \frac{\partial}{\partial w}\right)\right]_{(w, z)}=i \Gamma \frac{\partial}{\partial w}+i \beta z \frac{\partial}{\partial z}
$$

où $\beta \in \mathbb{R}$.

On a

$$
\left[f_{*}\left(i \frac{\partial}{\partial w}\right)\right]_{f(w, z)}=i \Gamma \frac{\partial}{\partial w}+i \frac{\partial f_{2}}{\partial w} \frac{\partial}{\partial z}
$$

d'où

$$
\beta f_{2}(w, z)=\frac{\partial f_{2}}{\partial w}(w, z)
$$

On déduit alors de (43) que $f_{2}(w, z)=A(z) e^{\beta w}$ où $A$ est une fonction entière telle que $A(0)=0$ et $A^{\prime}(0) \neq 0$. Montrons maintenant que $\beta=0$, 
nous procédons par l'absurde, supposons $\beta \neq 0$, d'parés le théorème 2 de [2], on a $P_{2}(z, \bar{z})=P_{2}(|z|,|z|)$ ou encore: $P_{2}(z, \bar{z})=\sum_{j=m}^{M} \alpha_{j}|z|^{2 j}$ avec $\alpha_{m}>0$ et $\alpha_{M}>0$.

L'inclusion $f\left(b \Omega_{1}\right) \subset b \Omega_{2}$ se traduit par l'identité:

$$
\sum_{j=m}^{M} \alpha_{j}\left|A(z) e^{--\beta P_{1}(z, \bar{z})}\right|^{2 j}=\Gamma P_{1}(z, \bar{z})
$$

En écrivant $P_{1}(z, \bar{z})$ sous la forme

$$
P_{1}(z, \bar{z})=\sum_{l=m}^{M}|z|^{2 l} \operatorname{ReV}(z) \text {, où } V_{l}(z)=\sum_{j=0}^{N_{l}} \gamma_{j} z^{j},
$$

et en identifiant les termes de la forme $|z|^{2 m} z^{p}$ ou $|z|^{2 m} \bar{z}^{q}, \quad p, q \geq 0$, dans chacun des deux membre de (44), on voit facilement que $A^{m}$ est un polynôme.

Soit $\sigma \in \mathbb{C}^{*}$ un nombre complexe fixé tel que:

$$
\lim _{t \rightarrow+\infty} P_{1}(t \sigma, t \bar{\sigma})=+\infty
$$

( un tel $\sigma$ existe car $P_{1}$ est un polynôme sous-harmonique). en remplaçant $z$ par $t \sigma$ dans (44) et en faisant tendre $t$ vers $+\infty$ on voit que $\beta=0$.

Donc $f_{2}(w, z)=f_{2}(z)$. Traduisons maintenant l'inclusion $f\left(b \Omega_{1}\right) \subset b \Omega_{2}$ par:

$$
\Gamma P_{1}(z, z)=P_{2} o f_{2}(z)
$$

Nous terminons la preuve de i) en montrant que $f_{2}$ est un polynôme. si $f_{2}$ présnetait une singularité essentielle à l'infini alors il existerait une suite $\left(z_{n}\right)_{n \geq 0}$ telle que

$$
\lim _{n \rightarrow+\infty}\left|z_{n}\right|=+\infty \text { ct } f_{2}\left(z_{n}\right)=f\left(z_{0}\right) \text { pour tout } n
$$

Compte tenu de (45) la suite $P_{1}\left(z_{n}, \bar{z}_{n}\right)$ devrait être bornée et donc il exiserait $w_{0} \in \mathbb{C}$ tel que $\left(w_{0}, z_{n}\right)$ soit dans $\Omega_{1}$ pour tout $n$. Finalemont, la suite $f\left(w_{0}, z_{n}\right)$ serait constante ce qui est imposible puisque $f$ est finie. ainsi la fonction $f$ est polynômiale.

Passons maintenant à ii). On se place dans la cas iii) du théorème 2 de [2]. Le polynôme $P_{1}(z, \bar{z})$ est positif car si non il cxisterait $z_{0} \in \mathbb{C}^{*}$ tel 
que $P_{1}\left(z_{0}, \bar{z}_{0}\right)<0$ et $\left(\frac{i}{\lambda}, z_{0}\right)$ appartiendrait à $\Omega_{1}$ tandis que $f\left(\frac{i}{\lambda}, z_{0}\right)$ ne serait pas dans $\Omega_{2}$.

Considérons l'application holoporphe $F$ de $\Omega_{1}$ sur $\Omega_{2}$ donnée par:

$$
\begin{aligned}
F: \Omega_{1} & \longrightarrow \Omega_{2} \\
(w, z) & \longrightarrow\left(\Gamma w, f_{2}(w, z)(1+\lambda w)^{\frac{1}{m}}\right)
\end{aligned}
$$

Ainsi on est ramené au cas i). donc $F$ de la forme: $F(w, z)=(\Gamma w, g(z))$ où $g$ est un polynôme.

Ou cncore $f_{2}(w, z)=\frac{g(z)}{(1+i \lambda w)^{\frac{1}{m}}}$. L'inclusion $F\left(b \Omega_{1}\right) \subset b \Omega_{2}$ se traduit par: $P_{1}(z, \bar{z})=\frac{M I}{\Gamma}|g(z)|^{2 m}$.

\section{References}

[1] F.Berteloot., Attraction des disques analytiques et continuité holdérionne d'applications holomorphes propres, Banach Center Publications, Vol. 31, pp. $91-98,(1995)$.

[2] A.Chaouech., Auto-applications holomorphes propres des domaines polynomiaux rigides de $\mathbb{C}^{2}$. Publicacions Matematiqués. Vol. 40. $\mathrm{N}_{1}$, pp. $41-66,(1996)$.

[3] B. Coupet and S.Pinchuk., Holomorphic equivalence problem for weighted homogencous rigid donains in $\mathbb{C}^{n+1}$, Indiana University Math. Journal, to appcar.

[4] K.Oeljeklaus., On the automorplism group of ccrtain lyyperbolic domains in $\mathbb{C}^{2}$, Astérisque 217, pp. $193-216,(1993)$.

[5] N. Stanton., Infinitesinal CR automorphisms of rigid hypersurfaces in $\mathbb{C}^{2}$, Journal of Geometric analysis, vol.1, N.3, pp. $231-267$, (1991).

Received : March 10, 1998.

\section{Abdelaziz Chaouech}

Université Abdelmalek Essaâdi

Faculté des Sciences

Département de Mathématiques

B.P. 2121 Tétouan

MAROC 